Introduction MSM in particular HIV positive MSM have an increased risk of anal cancer. There is a paucity of data regarding anal HPV infection and high grade anal intraepithelial neoplasia (HGAIN) among MSM in Malaysia. The study is part of a larger regional collaboration with sites in Bangkok, Bali and Jakarta.

The aims of the study were to determine the prevalence, incidence and risk factors associated with high risk anal HPV infection and HGAIN among a cohort of MSM attending a screening site in Kuala Lumpur.

Methods 3 doctors received training in high resolution anoscopy (HRA). 52 MSM participants, 26 HIV positive and 26 HIV Negative, were enrolled into the study. Participants were screened at 3 time points - at baseline, 6 months and 12 months. Each participant completed a questionnaire on demographics, smoking and sexual history. Anal sample collection was then undertaken for liquid based anal cytology and anal HPV genotyping (Linear Array). This was followed by HRA and biopsy of abnormal areas. Patients with AIN 2 or 3 were offered treatment. Each participant was also screened for syphilis (RPR nad TPPA) at each visit.

Results The baseline results are presented. There were no anal cancers. The most common HPV genotype was HPV 16 in 17/ $52(33 \%)$ participants. 39 (75\%) had at least one high risk HPV infection. $26(50 \%)$ had abnormal cytology. 9/52 (17\%) had HGAIN (AIN 2 or 3). HGAIN correlated with high grade anal cytology (ASC-H or HSIL) in 6/9 patients. There was 1 newly diagnosed HIV infection. There were 6 incident syphilis infections. Demographic and sexual behaviour data and correlates of HGAIN will be presented.

Conclusion High risk anal HPV infection and HGAIN was highly prevalent at baseline within this cohort of Asian MSM reinforcing the importance of screening within this population. Disclosure of interest The study was funded by grant from TreatAsia.

\section{P10.04 A NEW PARADIGM FOR FOLLOW-UP OF MEN WITH ANAL SQUAMOUS CELL CANCER (ASCC)}

${ }^{1} \mathrm{~J}$ Langton-Lockton*, ${ }^{2} \mathrm{~K}$ Hesse, ${ }^{2} \mathrm{~A}$ Carr, ${ }^{2} \mathrm{AS}$ Field, ${ }^{1,2}{ }^{2} \mathrm{RJ}$ Hillman. ${ }^{1}$ Western Sydney Sexual Health Centre; ${ }^{2}$ St Vincent's Hospital, Sydney

\subsection{6/sextrans-2015-052270.432}

Introduction ASCC is the commonest non-AIDS defining cancer in HIV-positive men who have sex with men, with incidence rates of $>100$ cases $/ 100,000$. Five-year survival rates $(65 \%)$ are closely related to tumour size at time of diagnosis. Post treatment local recurrence is common (33\% of cases). Current follow-up protocols for local recurrence of ASCC are naked eye inspection and digital anorectal examination. Anal cytology and High Resolution Anoscopy (HRA) have been proposed as additional methods, as they have the ability to detect microscopic evidence of HPV-related lesions. We report findings following diagnosis of ASCC from a single hospital.

Methods Patients with ASCC who had at least one anal cytology test and/or HRA following initial diagnosis from 2001 to 2015 were identified. The HRA procedure includes an intra-anal swab for intraepithelial cytology, together with directly visualised HRA-guided anal biopsies for histopathology.

Results All 14 cases (13 intra-anal; 1 peri-anal) were male, with a mean age 56 years (44 to 68) at diagnosis, and 93\% were HIV positive. Typical ASCC treatment was chemo-radiotherapy
(86\%). Two patients had follow up anal cytology/HRA but had not received treatment for their ASCC at time of analysis.

The first HRA following treatment was at a median of 9 months (range 2-48) and median follow-up after treatment was 24 months (range 2-72); with subsequent HRA follow up at 6-12 month intervals.

The most significant cytology/histology findings at follow-up were high-grade squamous intra-epithelial lesions (HSIL - 36\%), low grade squamous intra-epithelial lesions (50\%), and 14\% completely negative. None had cytological or histological evidence of local ASCC recurrence.

Conclusion The use of anal cytology and HRA permit early identification of anal HPV-associated lesions, including HSIL. This has the potential to target those most at risk of ASCC recurrence. Further experience is needed to fully evaluate their role in long-term management.

Disclosure of interest statement No pharmaceutical grants were received in the development of this study.

\section{P10.05 COST-EFFECTIVENESS OF SCREENING FOR ANAL CANCER USING REGULAR DIGITAL ANO-RECTAL EXAMINATIONS IN HIV-POSITIVE MEN WHO HAVE SEX WITH MEN}

${ }^{1} \mathrm{OOng}^{*},{ }^{1,2,3} \mathrm{CK}$ Fairley, ${ }^{4} \mathrm{~S}$ Carroll, ${ }^{2} \mathrm{~S}$ Walker, ${ }^{2,3} \mathrm{M}$ Chen, ${ }^{2,3} \mathrm{~T}$ Read, ${ }^{2,3} \mathrm{C}$ Bradshaw, ${ }^{5} \mathrm{~A}$ Grulich, ${ }^{5} \mathrm{~J}$ Kaldor, ${ }^{1} \mathrm{P}$ Clarke. 'Melbourne School of Population and Global Health, The University of Melbourne; ${ }^{2}$ Melbourne Sexual Health Centre; ${ }^{3}$ Central Clinical School, Monash University; ${ }^{4}$ Chris O'Brien Lifehouse, Sydney; ${ }^{5}$ The Kirby Institute, University of New South UK

\subsection{6/sextrans-2015-052270.433}

Introduction The optimal screening strategy for early anal cancer detection in men who have sex with men (MSM) living with HIV is unknown.

Methods We estimated the cost-effectiveness of regular anal examinations to screen for anal cancer in HIV-positive men MSM living in Australia using a probabilistic Markov model. Data sources were based on the medical literature and a clinical trial of HIV-positive MSM receiving an annual anal examination in Australia. The main outcome measures were undiscounted and discounted (at 3\%) lifetime costs, life years gained, quality adjusted life years (QALY) gained and incremental cost-effectiveness ratio (ICER).

Results Base-case analysis estimated the average cost of screening for and management of anal cancer ranged from \$195 for no screening to $\$ 1,915$ for lifetime annual screening of men aged $\geq 50$ years. The incremental discounted, QALYs gained ranged from 0.02 for 4 yearly screening to 0.03 for annual screening of men aged $\geq 50$ years. Screening of men aged $\geq 50$ years generated ICERs of $\$ 29,760$ per QALY gained (for screening every 4 years), $\$ 32,222$ (every 3 years), and $\$ 45,484$ (every 2 years). Uncertainty for ICERs were most influenced by the cost (financially and decrease in quality of life) from a false positive result, specificity of the anal examination, the probability of detection outside a screening program and the discount rate.

Conclusion In settings where anal cytology for screening precursors of anal cancer is not available, screening for anal cancer by incorporating regular anal examinations into routine HIV care for MSM aged $\geq 50$ years is most likely to be cost-effective by conventional standards.

Disclosure of interest statement This project was funded by a program grant from the Australian National Health and Medical Research Council. 